\title{
EMOCIONES Y SUBJETIVIDADES JUVENILES EN TORNO A LA EXPERIENCIA EN LA UniversidAd InTERCUltural De ChIAPAs (UNICH)
}

\section{Youth EMOtIONS ANd AfFeCTIONS REgARding THEIR EXPERIENCES AT THE UniversidAd InTERCULtural De ChiAPAs (UNICH)}

\section{Claudia Elizabeth Ramírez-Domínguez* Tania Cruz-Salazar**}

\begin{abstract}
Resumen: En este trabajo se pretende mostrar cómo la experiencia educativa de los jóvenes en la Universidad Intercultural de Chiapas (UNICH) se traduce en una formación educativa desigual que ocasiona emociones desfavorables para el aprendizaje. Lejos del proyecto intercultural que apela a la interlocución y la convivencia, la experiencia universitaria resulta en descontento, apatía e individualismo, debido a la contradicción de las altas expectativas hacia a una educación universitaria alternativa y lo que experimentó el alumnado en la cotidianidad en el aula. Se usó el marco teórico de la subjetividad en la pedagogía, abordada a partir de las experiencias y emociones.
\end{abstract}

Palabras clave: educación superior, educación intercultural, calidad de la educación, proceso de aprendizaje, rendimiento escolar.

Abstract: The aim of this paper is to show that young people's educational experience at the Intercultural University of Chiapas (UNICH) is unequal, violent, and conflictive, thus negatively affecting students' emotions. Far from the intercultural education project promoting dialog and equal relationships, the university experience actually demobilizes students, pits them against one another, drives them away from community values, and encourages individualism. Our approach focused on subjectivity in pedagogy, using the categories of experiences and emotions. Our fieldwork demonstrated how the university, as an educational space, does not help to create or reproduce sociocultural integration, but rather promotes, at a very subjective level, perhaps unwittingly, conflict and disappointment.

Keywords: higher education, intercultural education, educational quality, learning process, academic achievement.

* Claudia Elizabeth Ramírez Domínguez. Candidata a doctora en Ciencias por El Colegio de la Frontera Sur, México. Temas de especialización: educación, psicología educativa. Correo electrónico: psicoterapiaexpansiva@gmail.com. ORCID: 0000-0002-3997-7527.

** Tania Cruz Salazar, doctora en Antropología Social por el Centro de Investigaciones y Estudios Superiores en Antropología Sociales (CIESAS), sede Ciudad de México, México. Investigadora titular A en El Colegio de la Frontera Sur, México. Tema de especialización: juventud, cambio sociocultural y migraciones indígenas. Correo electrónico: tcruzs@ecosur.mx. ORCID: 0000-0002-7714-3275.
Enviado a dictamen: 18 de junio de 2018 Aprobación: 11 de octubre de 2018 Revisiones: 1 


\section{Antecedentes y contexto}

L a educación formal sigue siendo una de las grandes apuestas para generar cambios en la sociedad; en este sentido, Pérez (2008) afirma que desde diferentes corrientes teóricas -el constructivismo, el liberalismo, la interculturalidadse ha aceptado que la función principal que la sociedad delega en la escuela es la preparación de las nuevas generaciones para su incorporación futura al mundo laboral. Los desacuerdos en torno a la función que ésta debe cumplir surgen cuando se trata de explicar qué significa el proceso de escolarización, cómo se realiza dicho proceso y los efectos que se generan en las personas que lo atraviesan. En México, esta función cuestionó la institucionalización de la interculturalidad después del año 2000. A partir de ese momento se inauguraron universidades interculturales en todo el país con el objetivo de atender la disfuncionalidad de la universidad tal y como había sido instaurada en el medioevo europeo y presentando como una alternativa el diálogo parejo entre las culturas diversas de México que habían sido desplazadas y minusvaloradas por la cultura mestiza hegemónica. Como respuesta gubernamental al movimiento armado del Ejército Zapatista de Liberación Nacional, estas universidades pretendieron en su inicio disminuir el abatimiento de la población indígena debido al rezago y la inequidad en el acceso al sistema educativo (Mateos y Dietz, 2016). Es en este contexto donde surge la Universidad Intercultural de Chiapas (UNICH) con la misión de abrir espacios de inclusión a la diversidad cultural y así reducir la inequidad educativa, teniendo como objetivo la formación de estudiantes críticos, reflexivos Y capaces de transformar la realidad de las comunidades indígenas, al vincular lo aprendido en el aula con las comunidades (Casillas y Santini, 2006).

De acuerdo con Bermúdez (2017), a partir de estas reformas en las políticas de inclusión en la educación superior también se experimentó un incremento en las investigaciones ligadas a la educación superior de indígenas. Esta autora afirma que dichos estudios están relacionados con las experiencias de vida, los aprendizajes y los desafíos no sólo académicos, sino familiares y personales, que viven las y los estudiantes en su trayectoria universitaria. En algunas investigaciones como las de Sartorello y Cruz (2013), Bastiani et al. (2012) y Ortelli y Sartorello (2011) se discuten las experiencias marcadas por tensiones o impactos interculturales debidos a la competitividad y a la lógica occidental mestiza imperante en la dinámica educativa.

Otras investigaciones están enfocadas en las identidades juveniles indígenas y en jóvenes que cursan estudios universitarios (Tipa, 2018; Cruz y Ortiz, 2013; Gómez, 2010), en situaciones relacionadas con la desigualdad de género (Tipa, 2018; Bermúdez, 2017) o bien en las reconfiguraciones identitarias después de cursar los programas de educación superior (Gallegos, 2017). Las investigaciones en torno a la experiencia de la UNICH han sido cruciales para entender los conflictos socioculturales que atraviesan las y los jóvenes indígenas al llegar a la universidad en la ciudad de San Cristóbal de Las Casas (Cruz y Ortiz, 2013), así como para entender los significados que adquieren la escolaridad y la profesionalización de dichos estudiantes (Czarny, 2016).

De la experiencia escolar de jóvenes mestizos en las universidades interculturales no hemos registrado estudios, y menos sobre su experiencia emotiva en el proceso de enseñanza-aprendizaje al interactuar con estudiantes de pueblos originarios. Por este motivo, Casillas Chain y Jácome (2007) afirman que la institución universitaria es indiferente a los procesos y cambios internos que atraviesan las y los estudiantes, de tal manera que tanto padres, como docentes y directivos, desconozcan lo que viven y sienten; si acaso, tienen una ligera idea de lo que construyen cognitiva y emocionalmente.

La confluencia de perspectivas desde donde se debaten los efectos del proceso de escolarización ha provocado distintas acciones gubernamentales para enfrentar esta problemática en Chiapas, así como para aumentar la oferta educativa; en esta dirección, se han abierto nuevas carreras y universidades sin profundizar en problemas de fondo que atañen al proceso enseñanza-aprendizaje. La reformulación de 
políticas educativas para tratar de alcanzar la supuesta coherencia sociocultural y resolver los conflictos académicos justificó, después de 1994, la apertura de la licenciatura en Gestión y Autodesarrollo Indígena en la Universidad Autónoma de Chiapas (UNACH) y las licenciaturas en Desarrollo Sustentable, Turismo Alternativo, Lengua y Cultura, y Comunicación Intercultural en la UNICH.

Paralelamente a la creación de dichos proyectos, existen investigaciones realizadas en esos contextos educativos que dan cuenta de la falta de sentido, la dispersión, la pérdida de interés o el entusiasmo que el estudiantado manifiesta hacia los saberes escolares occidentales (Saiz y Maldonado, 2009), así como la necesidad de cambiar la función práctica de la educación formal debido a las necesidades actuales de la juventud (Pérez, 2008; Díaz, 2006). En este sentido, Pérez (2008) argumenta que la escuela en sí misma se encuentra ante demandas contradictorias en el proceso de socialización de las futuras generaciones. Por un lado, se ve obligada a promover un "buen" desarrollo cognitivo enseñando comportamientos que permitan a la juventud incorporarse de manera eficaz a la sociedad, entendida ésta como el ámbito de la libertad de consumo, de elección y participación política, de la libertad y responsabilidad en la esfera de la vida familiar, así como de producción laboral exhaustiva; pero, por otro lado, éste es un perfil muy distinto al que se requeriría para su incorporación y su permanencia sumisa y disciplinada en el espacio escolar y en el mundo del trabajo asalariado.

\section{Perspectiva teórica y estrategia metodológica}

La subjetividad en la pedagogía es un tema poco trabajado, más si nos centramos en lo emocional como un asunto crucial en el proceso formativo de cualquier estudiante; desde dicha perspectiva se ven las emociones que surgen en el proceso cognitivo como base de la conciencia y la razón. Con la guía de otra persona, el conocerse a sí mismo y transformar el entorno resulta un aprendizaje lleno de sentimientos favorables. Lo vivido e introyectado en la relación pedagógica debiera encaminarse a la reconstrucción subjetiva, mientras que el conjunto de emociones intensamente experimentadas para cada una de las situaciones y los contextos particulares tendría que ser agradable. Las emociones son la base sobre la que se sustentan los procesos cognitivos, pues coadyuvan a almacenar y a recordar memorias de manera más efectiva, de tal manera que emoción/cognición es un binomio indisoluble, de ahí la importancia de comprenderlas en el proceso de encauzar, promover y generar aprendizajes significativos (Mora, 2013; Ibáñez, 2001). Al respecto Maturana (2018) afirma que todo sustento racional y toda acción humana tienen un fundamento emocional, por lo que las disposiciones corporales van en función de lo que vivimos. Estudiar las experiencias y sus emociones ayuda a entender los recuerdos y los aprendizajes que dan sentido a nuestra formar de actuar y ver el mundo. Ibáñez (2001) muestra en su investigación con mil estudiantes de pedagogía cómo el interés, la alegría, el entusiasmo y la satisfacción, así como la rabia, la impotencia, la inseguridad y el miedo, son emociones favorables y desfavorables que surgen en el proceso de aprendizaje. Al respecto, Casassus (2007, 2008) señala que el tipo de relación emocional de las personas con el conocimiento cobra vital importancia para llevar a buen término el aprendizaje, por lo que en nuestro estudio sobre las experiencias universitarias no habrían de obviarse las relaciones docente/estudiante y estudiante/estudiante ni las situaciones ocurridas en el espacio áulico, ya que todas contextualizan el gusto o el disgusto por los contenidos de la currícula, así como la comodidad o incomodidad de las interacciones encaminadas al aprendizaje.

Las experiencias universitarias y las emociones son referentes cruciales para el caso que aquí nos ocupa. El contexto universitario de la UNICH es un medio para la interacción y el incremento de repertorios no sólo por el hecho de ser un campo abierto y plural, sino por acoger a jóvenes de diversos pueblos originarios y promover su interacción (Sartorello y Cruz, 2013; Baronnet, 2016; Dietz, 2012). Es a través de estos contactos y relaciones socioculturales como se generan alianzas, confrontaciones y conflictos culturales. Nos interesa 
ver cómo esto se vive a nivel individual en el aula y cómo los procesos de enseñanza-aprendizaje logran o no su cometido. El estudiante, en tanto sujeto juvenil, cuenta con un espacio que lo incluye y lo excluye, que lo violenta o lo desorienta, que lo acoge o lo reta; un lugar que se vuelve cotidiano e íntimo. Ya que el espacio universitario resume la rutina diaria del y la joven y de sus compañeros de clase, se observa cómo algunas veces la dislocación, el desfase o lo impersonal hacen que el asidero identitario se vuelva una trinchera y no un puente, en donde el yo cultural juega un rol defensivo y de aislamiento en términos de diferencia (Appadurai, 2001). En este escenario, el modelo educativo, la práctica docente y los contenidos curriculares conducen a la siguiente pregunta: ¿cómo estas experiencias universitarias impactan en la subjetividad de un grupo de jóvenes estudiantes universitarios? Intentamos responder a dicho cuestionamiento ofreciendo una lectura sobre la subjetividad de los jóvenes estudiantes de la UNICH enfrentados al reto de transitar con éxito por la educación superior.

Con base en una metodología cualitativa, la estrategia que diseñamos se enfocó en el sujeto, sus experiencias y sus emociones. El método etnográfico nos permitió recuperar sus perspectivas a través de sus testimonios, que fueron recogidos en el aula, un espacio social universitario altamente dinámico que, al observarlo cotidianamente, logramos describir a profundidad (Geertz, 1988; Hammersley y Atkinson, 1994). Las técnicas de recolección de datos fueron la observación participante, las entrevistas semiestructuradas y las conversaciones informales, métodos pertinentes para la investigación educativa con la que se busca indagar sobre asuntos interpersonales cruzados por relaciones de poder (Bertaux, 2005; Vela, 2004). La entrevista fue el método privilegiado para la producción del relato del actor y sus emociones, la descripción densa sirvió para contextualizar la producción de los espacios sociales y sus relaciones. Se conformó un grupo de doce estudiantes de la licenciatura en Desarrollo Sustentable de la UNICH dispuestos a participar en la investigación, quienes relataron y reconstruyeron su trayectoria educativa desde el ingreso a la universidad, hasta el momento del estudio (ver Tabla 1). Así, mediante un consistente trabajo de campo de 2009 a 2010, nos dimos a la tarea de documentar la cotidianeidad educativa de este grupo de estudiantes.

Con el objetivo de ilustrar las trayectorias educativas de modo lineal y transversal, operamos los conceptos centrales, experiencias y emociones de modo individual y colectivo sin distinción de género, clase o etnia. Así, la sistematización y el análisis de datos se analizaron de acuerdo con: 1) situaciones, 2) creencias, 3) valores, 4) estímulos y 5) emociones, reportados en los relatos de las personas entrevistadas. Apostamos por la intersección de las condiciones socioeducativas en los testimonios registrados, detectando emociones - miedo, enojo, tristeza, decepción, orgullo, alivio y alegría - asociadas a momentos claves en su trayectoria educativa como los siguientes: 1) experiencias durante la formación universitaria; 2) dinámicas en el aula y relación docentesestudiantes y 3) dinámicas en el aula y la relación estudiantes-estudiantes. A partir de este análisis, presentamos los hallazgos en la siguiente sección con tablas interpretativas, discutidas posteriormente según los argumentos centrales planteados. En los testimonios se confirma que la meta en la vida de los entrevistados era estudiar una licenciatura, por lo que su ingreso a la universidad estuvo permeado por amplias expectativas e ilusiones tanto individuales, como colectivas. Los hallazgos muestran a jóvenes deseosos de una educación humanizada, basada en el establecimiento de lazos afectivos y horizontales con los docentes, así como entre el grueso de la comunidad estudiantil. El estudio revela que existe cierta diferencia de comportamiento y sentir entre los estudiantes provenientes de la ciudad y los de las comunidades rurales o indígenas: los procedentes de estas últimas comunidades, aunque no dejan de expresar inconformidad de diversas formas, presentan un mayor interés por la dinámica establecida en el aula y mayor respeto a los profesores y autoridades universitarias, lo cual asociamos con la posición social inferior en que la sociedad colonial situó a los indígenas y su mundo rural, exigiéndoles un trato de respeto sumiso hacia las figuras de autoridad urbanas, modalidad que ha sido heredada por la juventud indígena rural del mundo actual. 
En el caso de los estudiantes indígenas, las experiencias de discriminación y de sufrimiento estuvieron permeadas por el doble imaginario de alcanzar otro nivel de vida en términos de clase y de etnia (Stavenhagen, Montiel y Gall, 2001). El hecho de estudiar en la universidad los colocaba en una posición de mayor prestigio dentro de su comunidad, por lo que la categoría de estudiante universitario tenía alcances que iban más allá del espacio académico, circunstancia que tiene larga data en la historia de Chiapas (Pineda, 1995; Bourdieu, 1988).

\section{El antes y el durante de la vida como estudiante universitario}

En primer lugar, antes de entrar a la universidad el valor que los jóvenes otorgan a la educación formal es muy alto. Los hallazgos demuestran que esto es más evidente entre los estudiantes provenientes de comunidades rurales que entre los del medio urbano. Sobresalen las experiencias educativas previas - secundaria o preparatoriacargadas de maltrato, sarcasmo y ridiculización por parte de docentes y de compañeros no indígenas hacia los indígenas. La figura de autoridad del docente se basa en el conocimiento legitimado y en una posición de superioridad que la clásica educación tradicionalista ha otorgado al privilegiar el conocimiento académico y relegar cualquier otro. En la Tabla 2 se exponen las experiencias estudiantiles clasificadas mediante una graduación, de lo negativo a lo positivo, pasando por situaciones neutrales, agrupadas de acuerdo con el conocimiento o desconocimiento lingüístico y las asociaciones con la identidad étnica (ver Tabla 2).

En segundo lugar, las experiencias durante la realización de los estudios universitarios provocan que las altas expectativas se conviertan en serias decepciones. Durantelos años de formación universitaria, la cotidianidad en el aula de los estudiantes, las relaciones interétnicas y los nuevos términos del proceso enseñanzaaprendizaje hacen que experimenten situaciones poco agradables. Opinar regularmente resulta una habilidad en la que han experimentado poco antes de entrar a la universidad; analizar, reflexionar y debatir, tanto textos académicos como problemáticas actuales, también son experiencias nuevas porque, a diferencia de la educación tradicional como marco educativo, en la UNICH se plantea el construccionismo como modelo, aunque tampoco éste llegue a buen término.

En la Tabla 2 puede observarse cómo la formación educativa en la UNICH va generando desencanto ya que para la mayoría las creencias en una buena educación estaban asociadas con la exigencia, el compromiso del profesoradoy la buena calidad educativa, situaciones que extrañaron desde los primeros años en la universidad. A pesar de esta emoción, los estudiantes no se dieron de baja gracias a que la motivación inicial al ingresar a la universidad fue extremadamente fuerte y positiva, por lo que la esperanza permaneció como motivo para dar continuidad al proyecto universitario.

En algunos relatos pudimos observar que las y los estudiantes tenían conciencia crítica acerca de la calidad académica y ética del profesorado; sin embargo, terminaron por aceptar el limitado aporte teórico que éste les brindó en las clases. Otros afirmaron que deseaban estudiar algo distinto, pero que al final se conformaron con la expectativa de mejorar su nivel de vida y restaron importancia a la carrera. La certificación y el título, entonces, se volvieron los objetivos más importantes. Muchos escogieron la carrera de Desarrollo Sustentable por las posibilidades de trabajo que ésta ofrecía al finalizar los estudios profesionales.

En otros relatos fue claro el desinterés que mostraron hacia el tipo de educación que estaban recibiendo: "de mala calidad", "poco exigente" y "muy desigual". Deducir que la cotidianidad en el salón de clases era una pérdida de tiempo fue para muchos un triste hallazgo que los llevó a la desconexión del proceso enseñanza-aprendizaje como respuesta; de esta manera, normalizaron la falta de sentido o pérdida de tiempo en el aula.

\section{Dinámica en el aula y relación entre estudiantes y profesores}

La idea generalizada de que la escuela sólo coadyuva a desarrollar el raciocinio y que los mejores profesores son 
aquellos que dominan ciertas materias o muchas, no se percibe en la voz de las y los estudiantes entrevistados, pues al preguntarles sobre las características de sus mejores docentes, muchos hablaron de la afectividad como la mayor virtud. Fueron los docentes que en algunos momentos se preocuparon por ellos, y no los que dominaban los contenidos de la materia, los que recibieron su reconocimiento por ayudarles a crear nuevos significados y sentidos en sus experiencias como estudiantes. Nos parece que la necesidad de ser atendido y escuchado emocionalmente por el docente es un elemento central y constante en los testimonios.

En cuanto a la dinámica en el aula y a la relación entre docentes y estudiantes mencionaron el cansancio y el aburrimiento como emociones vinculadas con una dinámica poco constructiva, que consistía fundamentalmente en que el profesor protagonizaba monólogos, mientras que los estudiantes permanecían pasivamente sentados durante más de dos horas en cada clase escuchándole, sin tener la oportunidad de participar activamente. La necesidad de ser tomados en cuenta por parte de sus profesores fue una constante, por lo que varios solicitaron participar y esto se les fue negado. En el caso analizado, interpretamos esta situación como imposición y detención del poder (Foucault, 1992) por parte del docente, quien se concibe a sí mismo como transmisor de conocimientos a la manera del modelo tradicionalista, no construccionista (Casassus, 2008).

El sentimiento de decepción fue reportado por los estudiantes entrevistados, el cual asociaron tanto a la institución educativa, como a la formación académica y a la práctica docente, ante lo que mostraron mucho disgusto. Pese a ello, optaron por no intervenir; en este sentido, pueden considerarse el conformismo y la apatía expresiones de la seria inconformidad con que vivieron su trayectoria universitaria. Frente a las críticas, sugerencias u opiniones respecto a las incongruencias o a la mala calidad de la enseñanza en el aula, las respuestas del profesorado y de la institución fueron principalmente la sordera y la invisibilización de estas problemáticas, por lo que comúnmente se termina cediendo la razón a los docentes, lo que al mismo tiempo reproduce el desánimo en el estudiantado, que se ve empujado a una completa renuncia en sus deseos de querer hacer algo diferente. Debido a que las voces estudiantiles son constantemente silenciadas, la universidad envía un mensaje jerárquico e impositivo sobre los estudiantes, a quienes les queda claro que no tienen una opinión válida en su propia institución educativa. Algunos testimonios se muestran en la Tabla 3 (ver Tabla 3).

Las voces silenciadas y las experiencias de desmotivación institucionalizan un círculo vicioso en el que la educación formal impone la repetición y la normalización de la pasividad, porque ante los menores intentos de rebeldía o ruptura frente el statu quo en el espacio áulico, toma lugar la represión por parte de los profesores, ya que la opinión crítica se percibe como una acción subversiva. Esto provoca que los estudiantes sigan siendo receptores de lo que el docente dice, sin la posibilidad de cuestionarlo o criticarlo. Los testimonios en los que se refirieron emociones de admiración y respeto se basaban en la simpatía que los profesores mostraron por el bienestar del alumnado.

\section{Dinámica en el aula y relación entre los estudiantes}

Respecto a la dinámica relacional entre las y los estudiantes dentro del aula, encontramos diversas emociones tanto negativas como positivas; las primeras son el desánimo y el enojo asociados con la conflictividad y la rivalidad estudiantil, y las segundas, más favorables ante el aprendizaje, fueron el orgullo y la satisfacción, presentes principalmente entre los jóvenes indígenas participantes de esta investigación. Las diferentes emociones se explican por las identificaciones étnicas de los estudiantes, de manera que los mestizos expresaron más disgusto frente a la experiencia universitaria, mientras que los indígenas - en su mayoría chicos y chicas rurales de familias pobres con pocas posibilidades de estudiomencionaron sentirse orgullosos y comprometidos con el proceso de aprendizaje. Otra diferencia entre los jóvenes indígenas y mestizos está relacionada con el uso del tiempo libre, pues los primeros mencionaron 
que lo destinaban a trabajar, pues para ellos era indispensable ganar dinero para sostener su vida en la ciudad y estudiar. En este sentido, el imaginario del tiempo libre no lo visualizan de la misma manera que sus compañeros mestizos, en tanto que los primeros tienen la idea de aprovechar el tiempo lo más posible. Los diferenciales aquí son las condiciones étnica y de clase social. De igual manera, se muestra explícitamente que existe un mayor compromiso por parte de los estudiantes indígenas hacia las actividades académicas, para comprender y entender su mundo universitario, ya que ponen mayor énfasis en el presente universitario y en sus proyecciones hacia el futuro. Este sentido de vida les conecta en un primer momento con su valía como personas, les devuelve un valor negado por la sociedad por el hecho de ser indígenas, un valor que les lleva a luchar para alcanzar un lugar y mantenerse, lo que al mismo tiempo les hace proyectarse hacia el futuro en un empleo que les permita alcanzar un mejor nivel económico. En el caso de los estudiantes mestizos, encontramos que ellos sí disponían de un tiempo libre después de clases, lo que les colocaba en un lugar completamente diferente al de sus compañeros indígenas. Algunas de sus expresiones se incluyen en la Tabla 4 (ver Tabla 4).

Por otra parte, se encontró que algunos estudiantes lograban ver la falta de involucramiento que la mayoría mostraba en la realización de las actividades académicas; sin embargo, esa comprensión de la dinámica en el aula tampoco les motivaba para llevar a cabo acciones o intentar cambiar su cotidianidad en la universidad. En otras narraciones se encontró que asumían indiferencia y apatía hacia las clases y las actividades académicas porque cursaban una carrera que no eligieron. Ante este discurso, podría pensarse que, si las y los jóvenes estudiaran la carrera que realmente les gustara, mostrarían un mayor interés e involucramiento en su proceso de aprendizaje universitario.

En otros relatos se observa algo más profundo, que va más allá de la elección de la carrera por intereses propios o no; se trata de una desconexión de su proyección desde el presente y hacia su futuro como profesionistas. Pareciera que al llegar a la universidad empiezan a buscar elementos que les den mayor sentido a su vida actual, lo que de alguna manera desencadena la puesta en marcha de aquellos ideales en torno a los estudios universitarios, al mismo tiempo que se ponen en juego las emociones de inseguridad y de valía, por lo que constantemente entran en comparación con sus compañeros, lo que hace emerger sentimientos de envidia o impulsa acciones de imitación aunque lo que haga el otro no les ayude a consolidar su formación académica universitaria; por ello, se reproducen acciones de ausencia en clases, que justifican mencionando que eso es lo que hacen sus compañeros y porque saben que, a pesar de sus inasistencias, podrán acreditar sus materias y el semestre.

Otro momento en el que se vio reflejada esa repetición de conductas y el apaciguamiento de su proceso de aprendizaje en la universidad fue en la dinámica instaurada en el aula en términos de participación, pues la mayoría de ellos se ha acostumbrado a la inacción en su salón de clases, a conformarse con asistir y estar sentado en su silla, pues al final alguien participará y los eximirá de tener que activarse en las clases.

Otro aspecto relevante en las narraciones es la sensación de pertenecer a un grupo fragmentado, con poco espíritu de colectividad, lo que genera un ambiente de poca confianza. Al mismo tiempo, las y los estudiantes tienen la creencia de que existe una escisión entre el intelecto y la emoción, por lo que adjudican a la universidad la función de desarrollar habilidades únicamente cognitivas, dejando fuera las habilidades socioemocionales. De esta manera, a pesar de ver y sentir la desunión en su grupo, no consideran el aula como un espacio para desarrollar habilidades emocionales; así, la universidad es vista como el espacio para trabajar aspectos académicos, y sólo fuera de ella cada estudiante aprende a gestionar dichas habilidades socioafectivas.

\section{Conclusiones}

En los relatos de las y los jóvenes se observa una fuerte contradicción entre lo esperaban al entrar a la universidad, es decir, un proyecto de educación alternativo que plantea la formación de estudiantes 
críticos y dispuestos al diálogo intercultural (Casillas y Santini, 2006), y lo que experimentan en la cotidianidad en el aula, es decir, un alejamiento de aquel proyecto que los conduce a la pasividad y les provoca una desconexión entre su sentir desilusionante y su actuación en el salón de clases. En este sentido, los testimonios muestran a estudiantes que iniciaron sus estudios universitarios con mucho entusiasmo y con altas expectativas respecto a la UNICH, ya que esperaban encontrarse con un centro de estudios de alto nivel de exigencia que les formara como estudiantes competentes, con un alto rendimiento académico. Sin embargo, con el transcurrir del tiempo ese ímpetu académico se transformó en abandono del reordenamiento de significados y de sentidos para sí mismos. Los deseos iniciales de asistir todos los días a la universidad disminuyeron; si bien demandaban una enseñanza de alta calidad por parte de los profesores, al dejarles éstos con grandes vacíos en su aprendizaje, los alumnos perdieron el interés por estudiar y transformaron el proyecto universitario en uno de certificación para ingresar posteriormente al campo laboral. Las decisiones tomadas por los estudiantes, quienes entran en el juego de la apariencia al decidir continuar con la aburrida y de mala calidad trayectoria universitaria, en vez de cambiarse a otra universidad o exigir una mejora en la calidad, permite observar su rol de "víctimas del sistema educativo", sin llegar a empoderarse en el presente y a proyectarse en el futuro.

Los relatos de los jóvenes universitarios remiten a nociones y expectativas de movilidad social ascendente asociadas a la educación formal y, especialmente, a la superior. El ingreso a la universidad significa para muchos la excelencia académica y un cambio de panorama en la ejecución de este proyecto tanto individual, como colectivo. La familia comparte con ellos los ideales de la educación superior y deposita en los hijos o hijas estudiantes los anhelos de desarrollo intelectual, social y cultural que las generaciones previas no pudieron alcanzar. Llama la atención que las emociones más recurrentes fueron la decepción y el desencanto, emociones desfavorables para nuevos aprendizajes. El descuido institucional y docente puede explicarse como consecuencia de una visión tradicional de la educación, que obvia dimensiones pedagógicas centradas en el desarrollo y la fortaleza emocional del sujeto estudiante, quien ve el proceso de aprendizaje como repetición, memorización o estímulo-respuesta, y el foco de atención se pone en el maestro más que en el estudiante (Casassus, 2008).

Un aspecto relevante en la trayectoria académica universitaria es el reclamo de una formación educativa con mayor énfasis en el desarrollo de las habilidades emocionales, dado que en algunas historias los estudiantes conceden un lugar especial a la pedagogía experiencial (Keck y Saldívar, 2016); nos referimos a la importancia de enfocarse en el "proceso", más que en el resultado educativo, es decir, en aquellos aprendizajes que verdaderamente se quedan guardados en la memoria durante la realización de las actividades (Díaz, 2006). Este enfoque en el proceso implicaría que los docentes dedicaran más tiempo dentro del aula a desarrollar en sus estudiantes estrategias para el cuidado de sí mismos en un amplio sentido (Keck, 2018), pues las y los jóvenes consideran como sus mejores docentes a aquellos que tomaban más en consideración la emocionalidad del sujeto, ya que en su memoria quedaron registros de aquellos que concedían una dedicación significativa al cuidado de la persona y de las emociones, de modo que a través de ese vínculo afectivo podían contar con una mano amiga que les ayudara a resolver sus problemas personales. Esta habilidad que mostraron sus docentes para considerarlos como personas hacía que sintieran admiración y respeto por este tipo de profesores.

Las emociones que subyacen en las subjetividades de las y los jóvenes muestran la urgencia de trabajar en el aula tanto los aspectos cognitivos como los emocionales de manera transversal. De esta manera, el modelo educativo intercultural de la UNICH, al ser una propuesta alternativa y novedosa, debería poner mayor énfasis en una verdadera práctica de educación integral, lo que implica el desarrollo de habilidades socioemocionales tanto en los docentes, como en los estudiantes. 


\section{Referencias}

Appadurai, Arjun (2001). La modernidad desbordada. Dimensiones culturales de la globalización. México: FCE, Trilce.

Baronnet, Bruno (2016). "La educación intercultural crítica como construcción de los pueblos originarios en México". En Isabel de la Cruz Pastor, Humberto Santos Bautista y David Cienfuegos Salgado (eds.), Interculturalidad, conocimiento y educación. Diálogos desde el Sur. Chilpancingo, México: Universidad Pedagógica Nacional, El Colegio de Guerrero, pp. 179-202.

Bastiani Gómez, José, Lorena Ruiz Montoya, Erín Estrada Lugo, Tania Cruz Salazar y José Antonio Aparicio Quintanilla (2012). "Política educativa indígena. Práctica docente, castellanización, burocracia y centralización de la educación como limitaciones del éxito pedagógico en la región Ch’ol, Chiapas". En Revista Perfiles Educativos, 34(135): 8-25.

Bermúdez Urbina, Flor Marina (2017). "La investigación sobre trayectorias académicas y de estudiantes en la educación superior intercultural en México". En Revista Educación y Desarrollo Social, 11(1): 116-145.

Bertaux, Daniel (2005). Los relatos de vida. Perspectiva etnosociológica. Barcelona: Bellaterra.

Bourdieu, Pierre (1988). La distinción. Criterios y bases sociales del gusto. Madrid: Taurus.

Casassus Gutiérrez, Juan (2007). La educación del ser emocional. Chile: Índigo, Cuarto Propio.

Casassus Gutiérrez, Juan (2008). "Aprendizaje, emociones y clima en el aula”. En Paulo Freire, Revista de Pedagogía Crítica, 7(6), 81-95.

Casillas, Miguel, Ragueb Chain y Nancy Jácome (2007). "Origen social de los estudiantes y trayectorias estudiantiles en la Universidad Veracruzana". En Revista de la Educación Superior, XXXVI(2-142): 7-29.

Casillas Muñoz, María de Lourdes y Laura Santini Villar (2006). Universidad intercultural: modelo educativo. México: SEP-Coordinación General de Educación Intercultural y Bilingüe.

Cruz Salazar, Tania y Alfonso Ortiz Moreno (2013) "Juventudes interculturales. Voces y visiones estudiantiles en la UNICH". En Stefano Claudio Sartorello Tania Cruz Salazar (coords.), Voces y visiones juveniles en torno a la diversidad, diálogo y conflicto intercultural en la UNICH. México: Editorial Fray Bartolomé de Las Casas, pp. 65-92.

Czarny, Gabriela (2016). "Jóvenes indígenas y relatos sobre escolaridades en la Universidad Pedagógica Nacional". En Revista del Cisen Tramas/Maepova, 4(1): 137-151.

Díaz Barriga Arceo, Frida (2006). La enseñanza situada: vínculo entre la escuela y la vida. México: McGraw-Hill.

Dietz, Gunter (2012). Multiculturalismo, interculturalidad y diversidad en educación. Una aproximación antropológica. México: Fondo de Cultura Económica.

Foucault, Michel (1992). Elorden del discurso. Buenos Aires: Tusquets.

Gallegos Martínez, Gabriela (2017). Reconfiguración identitaria de jóvenes indígenas en contextos de migración. Tersis de maestría. ECOSUR, San Cristóbal de Las Casas, Chiapas.

Geertz, Clifford (1988). La interpretación de las culturas. Barcelona: Gedisa.

Gómez Díaz, Clara (2010). Estilos juveniles y consumo cultural en la Universidad Intercultural de Chiapas. Tesis de licenciatura, Universidad Autónoma de Chiapas, Chiapas.

Hammersley, Martyn y Paul Atkinson (1994). Etnografía. Métodos de investigación. Buenos Aires: Paidós.

Ibáñez, Nolfa (2001). "El contexto interaccional en el aula: una nueva dimensión evaluativa". En Estudios Pedagógicos, 27: 43-53.

Keck, Charles Stephen (2018). Ser docente, ser persona: once relatos de aprendizaje y transformación socioemocional. México: El Colegio de La Frontera Sur.

Keck, Charles Stephen y Antonio Saldívar (2016). "Una mirada a la formación docente desde la experiencia: una apuesta por el no-futuro de la educación". En Sinéctica, Revista Electrónica de Educación, 47: 1-19.

Mateos Cortés, Laura Selene y Gunther Dietz (2016). "Universidades interculturales en México: balance crítico de la primera década”. En Revista Mexicana de Investigación Educativa, 21(70): 683-690. Disponible en: http://www.redalyc.org/articulo.oa?id=14046162002. 
Maturana, Humberto(2008). Emociones y lenguajeeneducación y política. Material para el curso de Teoría de Sistemas. Extracto del texto Emociones y Lenguaje en Educación y Politica. S/l: CED. Disponible en: http://turismotactico.org/ proyecto_pologaraia/wp-content/uploads/2008/01/ emociones.pdf

Mora Teruel, Francisco (2013). "¿Qué es una emoción?". En Arbor, 189(759). DOI: http://dx.doi.org/10.3989/ arbor.2013.759nl003

Ortelli, Paola y Stefano Claudio Sartorello (2011). “Jóvenes universitarios y conflicto intercultural". En Perfiles Educativos, 3: 77-90.

Pérez Gómez, Ángel (2008). “Las funciones sociales de la escuela: de la reproducción a la reconstrucción crítica del conocimiento y la experiencia". En José Gimeno Sacristán y Ángel I. Pérez Gómez (coords.), Comprender y transformar la enseñanza. Madrid: Morata.

Pineda, Luz Olivia (1995). "Maestros bilingües, burocracia y poder político en Los Altos de Chiapas". En Juan Pedro Viqueira y Mario Humberto Ruz (eds.), Chiapas. Los rumbos de otra historia. México: UNAM, CIESAS, CEMCA, Universidad de Guadalajara, pp. 279-300.
Saiz, María Carolina y Mónica Maldonado (2009). "Los medios y el desinterés de los alumnos por el aprendizaje escolarizado". En Revista UIS Humanidades, 37(2): 71-89.

Sartorello, Stefano Claudio y Tania Cruz Salazar (2013). Voces y visiones juveniles en torno a diversidad, diálogo y conflicto intercultural en la UNICH. San Cristóbal de Las Casas, Chiapas: Editorial Fray Bartolomé de Las Casas. Stavenhagen, Rodolfo, L.M. Martínez Montiel y Olivia Gall (2001). "La mexicana, una sociedad racista". En Rebelión. Cultura, 5 de mayo. Disponible en: http:// www.rebelion.org/hemeroteca/cultura/mexicana racista09050l.htm

Tipa, Juris (2018). “iLa interculturalidad es más que una palabra? La interculturalidad según los estudiantes de la Universidad Intercultural de Chiapas". En Antrópica, Revista de Ciencias Sociales y Humanidades, 3(6): 17-34.

Vela Peón, Fortino (2004). "Un acto metodológico básico de la investigación social: la entrevista cualitativa". En María Luisa Tarrés (coord.), Observar, escuchar y comprender. Sobre la tradición cualitativa en la investigaciónsocial. México: Colmex, FLACSO, Porrúa, pp. 63-95. 
Tabla 1. Estudiantes que participaron

\begin{tabular}{|c|c|c|c|c|c|c|}
\hline & Nombre & Edad & $\begin{array}{l}\text { Mujer/ } \\
\text { hombre }\end{array}$ & $\mathrm{EC}^{*}$ & Procedencia & Lenguas \\
\hline 1 & Jesús & 22 años & Hombre & $\mathrm{S}$ & Chanal, municipio de Chanal & $\begin{array}{l}\text { Tseltal, } \\
\text { español }\end{array}$ \\
\hline 2 & Angélica & 21 años & Mujer & $S$ & Sacsalum, municipio de Las Margaritas & Español \\
\hline 3 & Isabel & 21 años & Mujer & $\mathrm{S}$ & $\begin{array}{l}\text { Lázaro Cárdenas, municipio de La } \\
\text { Trinitaria }\end{array}$ & Español \\
\hline 4 & José & 28 años & Hombre & $\mathrm{S}$ & San Juan Cancuc, municipio de Ocosingo & $\begin{array}{l}\text { Tseltal, } \\
\text { español }\end{array}$ \\
\hline 5 & Elías & 22 años & Hombre & $\mathrm{S}$ & $\begin{array}{l}\text { San Caralampio, municipio de La } \\
\text { Trinitaria }\end{array}$ & Español \\
\hline 6 & Félix & 23 años & Hombre & $\mathrm{S}$ & $\begin{array}{l}\text { San Cristóbal de Las Casas, municipio de } \\
\text { San Cristóbal de Las Casas }\end{array}$ & Español \\
\hline 7 & Ernesto & 24 años & Hombre & $\mathrm{S}$ & $\begin{array}{l}\text { San Cristóbal de Las Casas, municipio de } \\
\text { San Cristóbal de Las Casas }\end{array}$ & Español \\
\hline 8 & Roque & 29 años & Hombre & C & $\begin{array}{l}\text { San Cristóbal de Las Casas, municipio de } \\
\text { San Cristóbal de Las Casas }\end{array}$ & Español \\
\hline 9 & Gilberto & 25 años & Hombre & $\mathrm{S}$ & Tenejapa, municipio de Tenejapa & $\begin{array}{l}\text { Tseltal, } \\
\text { español }\end{array}$ \\
\hline 10 & Miguel & 21 años & Hombre & $\mathrm{S}$ & San Pedro, municipio de Chenalhó & $\begin{array}{l}\text { Tseltal, } \\
\text { español }\end{array}$ \\
\hline 11 & Gaby & 23 años & Mujer & $\mathrm{S}$ & $\begin{array}{l}\text { San Cristóbal de Las Casas, municipio de } \\
\text { San Cristóbal de Las Casas }\end{array}$ & Español \\
\hline 12 & Lili & 22 años & Mujer & $\mathrm{S}$ & $\begin{array}{l}\text { San Cristóbal de Las Casas, municipio de } \\
\text { San Cristóbal de Las Casas }\end{array}$ & Español \\
\hline
\end{tabular}

Fuente: elaboración propia.

* EC: estado civil. 


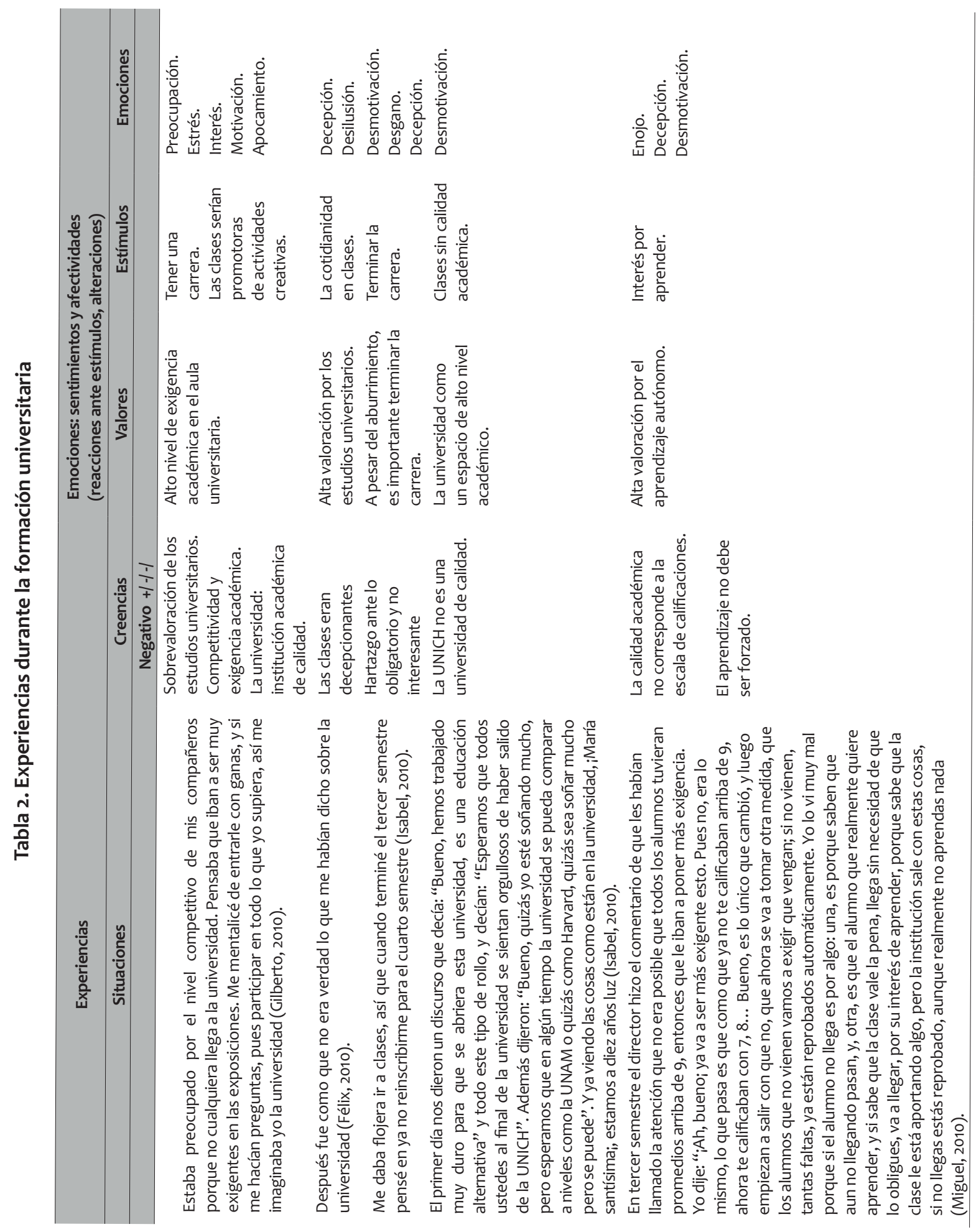




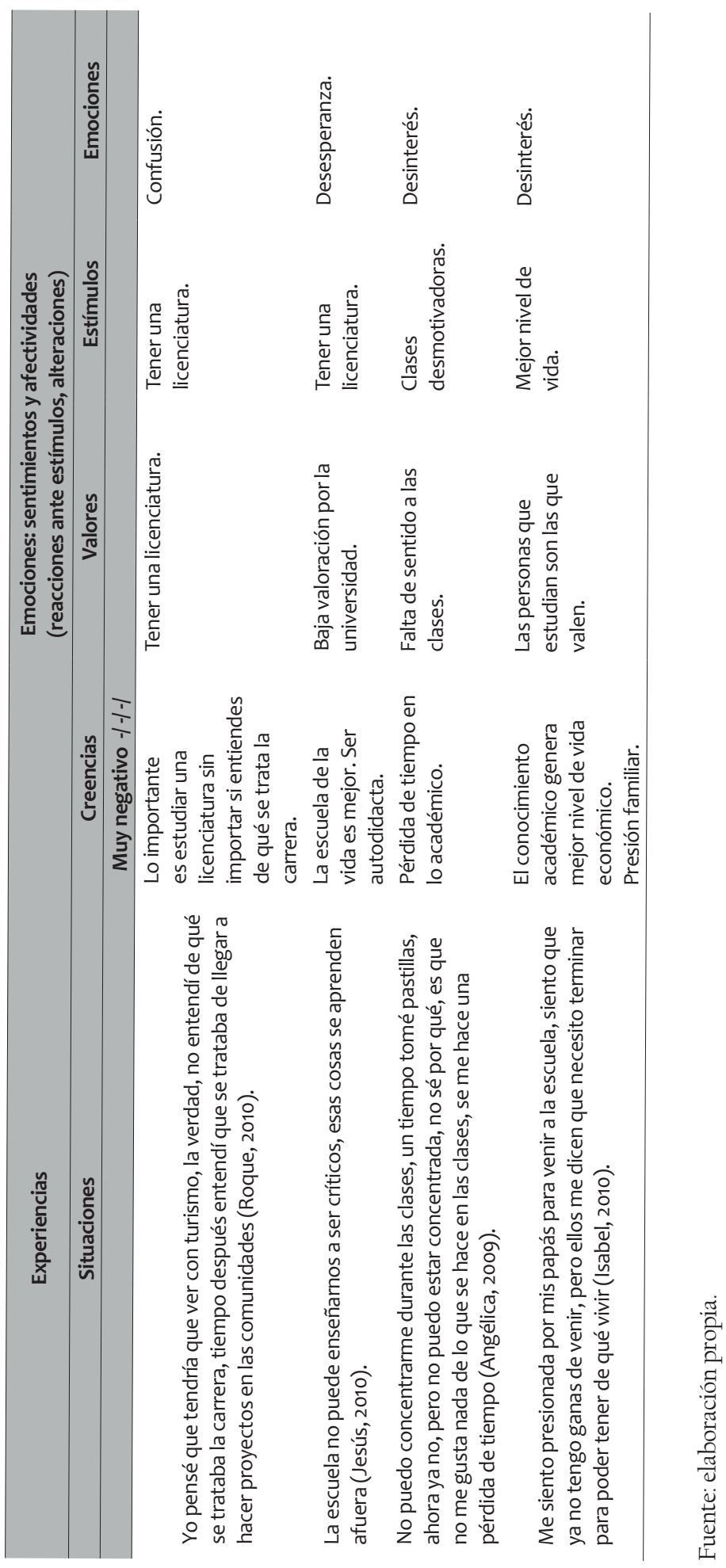




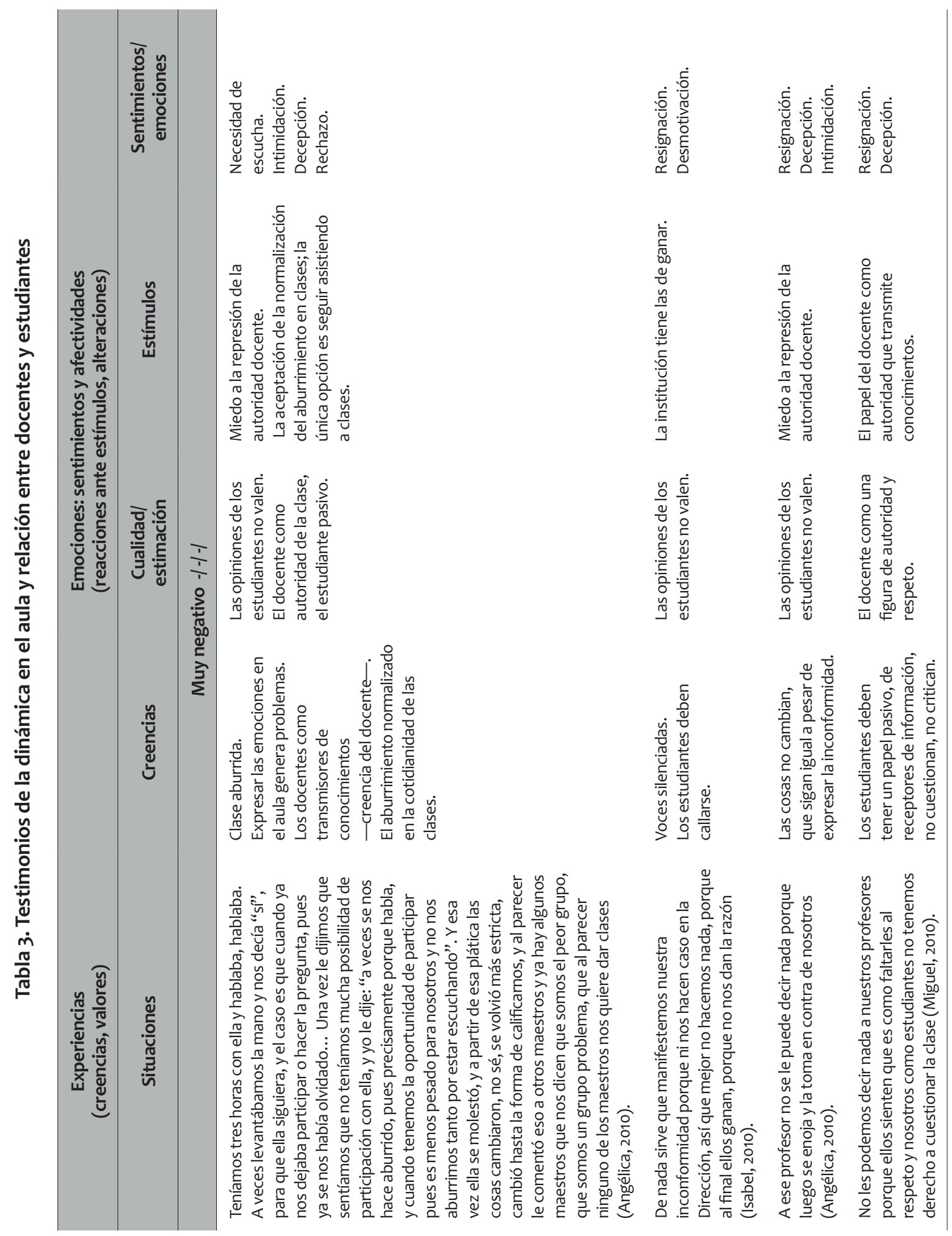




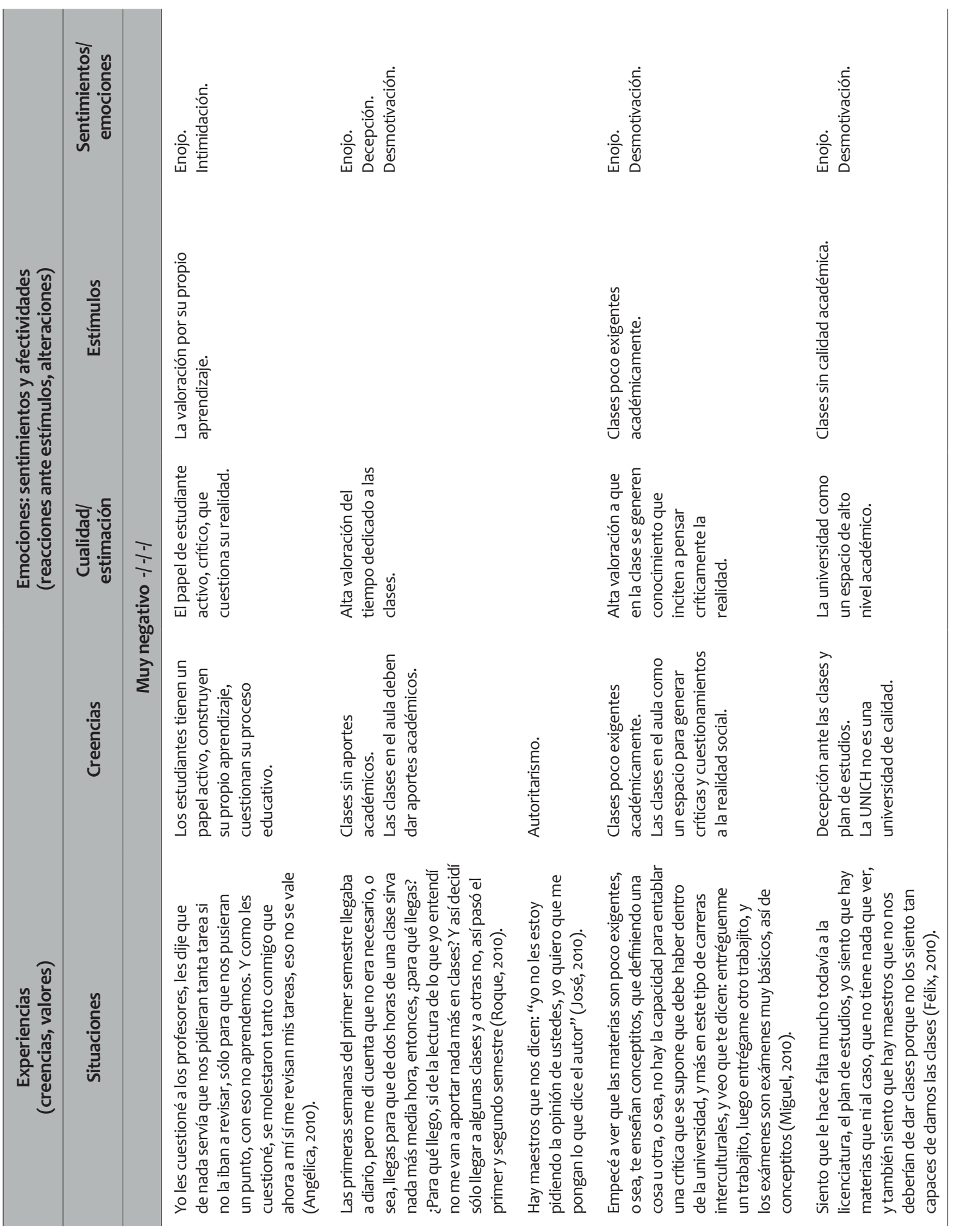




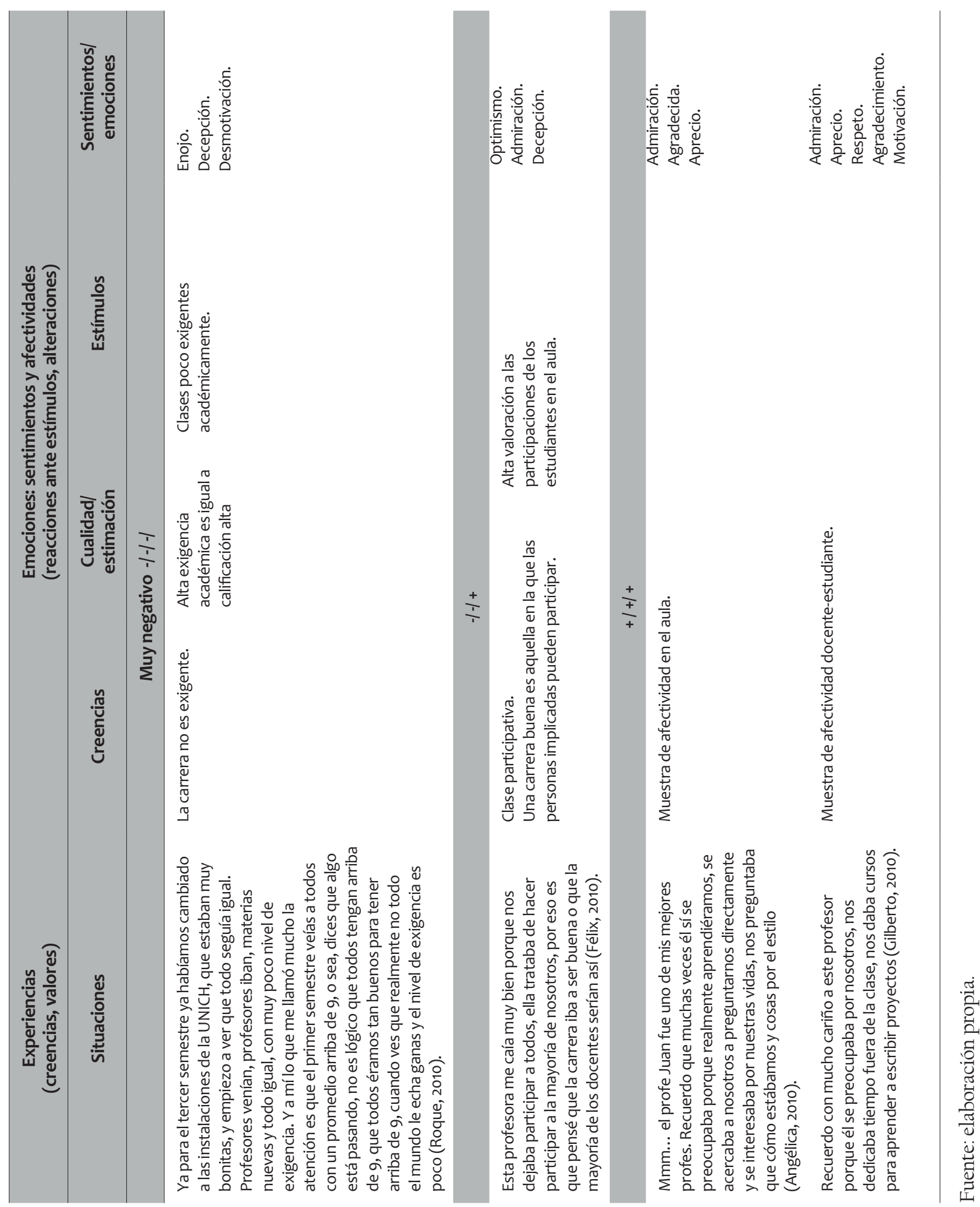




\section{Tabla 4. Dinámica en el aula y relación entre estudiantes}

$\begin{array}{cr}\text { Experiencias } & \begin{array}{l}\text { Emociones: sentimientos y afectividades } \\ \text { (reacciones ante estímulos, alteraciones) }\end{array}\end{array}$

Situaciones

\section{Creencias}

Cualidad/estimación

\section{$H-H$}

La verdad es que el grupo tampoco ayuda; si te das cuenta, acá la mayoría no se lleva entre sí, y eso también desanima porque no podemos ponernos de acuerdo para nada (Miguel, 2010).

Yo creo que a la mayoría de mis compañeros no les interesa venir a clases porque no eligieron esta carrera. Si les preguntas a todos mis compañeros te vas a dar cuenta que ellos querían estudiar otra cosa (Félix, 2010).

Yo siempre he dicho que son... que somos más chingones que los budistas, porque los budistas se llevan años para aprender a separar el cuerpo y la mente, cuando nosotros podemos estar en la escuela y somos un bulto en la butaca y la mente por otro lado (Roque, 2010).

\section{Poca afinidad de} carácter entre compañeros de clase. El grupo en sí mismo, es responsable de la confrontación.

Desinterés por la licenciatura. La licenciatura como un valor dentro de la sociedad.

Soñar despiertos en el aula.

Los estudiantes sólo llegan a hacer bulto a las clases.

\section{Generar afinidades}

de carácter

en el aula es

responsabilidad de

cada persona.

\section{La licenciatura como valor dentro de la sociedad, sin importar tus intereses sobre una carrera en específico.}

\section{Alta valoración} hacia las personas que dentro del aula están presentes en cuerpo y alma.

\section{Tener una} licenciatura sin importar qué estudiar.
Sentimientos/ emociones

\section{$-1-1$}

La escuela no podría hacer nada con la desunión del grupo, estos son problemas personales, y la escuela sólo debe ver los aspectos académicos, cada uno de nosotros tiene que poner de su parte para llevarse bien con los demás (Jesús, 2010).

\section{Desunión grupal.} La escuela sólo genera conocimientos intelectuales.

\section{Alta valoración a la} separación entre lo académico y lo afectivo en el salón de clases.

\section{Decepción.}

Desánimo. Necesidad de afecto.

Desinterés.

Resignación. Desmotivación.

\footnotetext{
-

La verdad es que ya sabemos quiénes son los que hablan siempre en la clase, entonces, cuando el profe pide una participación, pues todos esperamos a que ellos hablen, aunque hay uno que participa más, nosotros sabemos que "ese güey" va a decir algo. (Ernesto, 2010)

Pocas participaciones en el aula.

En un grupo es imposible que todas las personas participen.
}

Resignación. Necesidad de afecto.

Expectativa: escuela como generadora sólo de conocimiento.

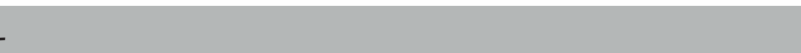




\begin{tabular}{|c|c|c|c|c|}
\hline \multirow{2}{*}{$\begin{array}{c}\begin{array}{c}\text { Experiencias } \\
\text { (creencias, valores) }\end{array} \\
\text { Situaciones }\end{array}$} & \multicolumn{4}{|c|}{$\begin{array}{l}\text { Emociones: sentimientos y afectividades } \\
\text { (reacciones ante estímulos, alteraciones) }\end{array}$} \\
\hline & Creencias & Cualidad/estimación & Estímulos & $\begin{array}{l}\text { Sentimientos/ } \\
\text { emociones }\end{array}$ \\
\hline $\begin{array}{l}\text { Me di cuenta de que, si habían pasado varios } \\
\text { compañeros sin hacer casi nada, entonces yo } \\
\text { también podía seguir llegando y no me darían de } \\
\text { baja (Roque, 2010), }\end{array}$ & $\begin{array}{l}\text { Las inasistencias en } \\
\text { clases, } \\
\text { Los estudiantes que } \\
\text { no llegan a clases } \\
\text { deben reprobar. }\end{array}$ & $\begin{array}{l}\text { Alta valoración } \\
\text { de la formación } \\
\text { académica } \\
\text { presencial. }\end{array}$ & $\begin{array}{l}\text { Las inasistencias } \\
\text { de los } \\
\text { compañeros. }\end{array}$ & $\begin{array}{l}\text { Enojo. } \\
\text { Decepción. } \\
\text { Desmotivación. }\end{array}$ \\
\hline \multicolumn{5}{|c|}{0} \\
\hline $\begin{array}{l}\text { La escuela no puede enseñarnos a ser } \\
\text { críticos, esas cosas se aprenden afuera. } \\
\text { (Jesús, 2010) }\end{array}$ & $\begin{array}{l}\text { Pensar críticamente } \\
\text { fuera del aula. } \\
\text { La escuela como } \\
\text { reproductor de } \\
\text { saberes intelectuales. }\end{array}$ & $\begin{array}{l}\text { Alta valoración por } \\
\text { los aprendizajes que } \\
\text { se generan fuera del } \\
\text { aula. }\end{array}$ & & Desesperanza. \\
\hline $\begin{array}{l}\text { Cuando regreso de la escuela a veces veo } \\
\text { televisión, pero a veces mi mamá me pide } \\
\text { que la acompañe al centro, entonces ya voy } \\
\text { con ella y mi tarea la hago en la escuela, total, } \\
\text { no importa mucho, jeje (Roque, 2010). }\end{array}$ & $\begin{array}{l}\text { Despreocupación. } \\
\text { Desinterés académico. } \\
\text { Desmotivación. }\end{array}$ & & & \\
\hline \multicolumn{5}{|c|}{$+/+$} \\
\hline $\begin{array}{l}\text { [...] me siento orgulloso de que por lo } \\
\text { menos los represento a ellos. Es que es } \\
\text { como ahorita, soy estudiante indígena tseltal } \\
\text { y soy del municipio de Chanal, y si hacen } \\
\text { aquí un diagnóstico, digamos, somos cinco } \\
\text { o diez muchachos, pero representamos } \\
\text { al pueblo, porque antes, unos diez años } \\
\text { atrás, no había ni un joven que saliera a } \\
\text { estudiar y menos que fuera universitario, lo } \\
\text { máximo que llegaban era la prepa. Siento } \\
\text { un compromiso primeramente hacia mí, } \\
\text { de ahí hacia mi familia y a todos los que me } \\
\text { han visto crecer. Porque me he encontrado } \\
\text { varios profesores de la primaria y me dicen: } \\
\text { “¿qué, estás estudiando la universidad?” Y les } \\
\text { digo: “Sí”, y les da mucho gusto, y como que } \\
\text { mis tíos o señores que me han visto crecer } \\
\text { en mi pueblo, y cada vez que los encuentro } \\
\text { me preguntan qué estoy haciendo; “qué } \\
\text { estás estudiando?”, y les comento, “estoy } \\
\text { estudiando esto y se trata de esto”, y } \\
\text { dicen: “pues qué bien, felicidades, échale } \\
\text { ganas, algún día... vas a ver... pronto vas a } \\
\text { funcionar para algo en el pueblo”. Entonces } \\
\text { quiere decir que por lo menos ya valgo más } \\
\text { porque me están tomando en cuenta (Jesús, } \\
\text { 2010). }\end{array}$ & $\begin{array}{l}\text { Cambio sociocultural. } \\
\text { Mejor preparación } \\
\text { académica en la } \\
\text { ciudad. }\end{array}$ & $\begin{array}{l}\text { Alta valoración } \\
\text { por los estudios } \\
\text { universitarios. }\end{array}$ & & $\begin{array}{l}\text { Orgullo. } \\
\text { Satisfacción. } \\
\text { Motivación. } \\
\text { Interés académico. }\end{array}$ \\
\hline
\end{tabular}




\begin{tabular}{|c|c|c|c|c|}
\hline \multirow{2}{*}{$\begin{array}{c}\begin{array}{c}\text { Experiencias } \\
\text { (creencias, valores) }\end{array} \\
\text { Situaciones }\end{array}$} & \multicolumn{4}{|c|}{$\begin{array}{l}\text { Emociones: sentimientos y afectividades } \\
\text { (reacciones ante estímulos, alteraciones) }\end{array}$} \\
\hline & Creencias & Cualidad/estimación & Estímulos & $\begin{array}{l}\text { Sentimientos/ } \\
\text { emociones }\end{array}$ \\
\hline \multicolumn{5}{|c|}{$+/+/+$} \\
\hline $\begin{array}{l}\text { Llevaba mis apuntes a mi trabajo, llevaba ahí } \\
\text { en ratitos, ahí me sentaba yo en las escaleras } \\
\text { a ponerme a leer aunque sea arrugado, pero } \\
\text { ahí estaban, en cualquier momento, y yo creo } \\
\text { que eso facilitaba o facilitó mi aprendizaje, } \\
\text { o sea, de que tiene que ser en cualquier } \\
\text { momento, hay que aprovechar el tiempo, } \\
\text { y al final como que se te facilita más, como } \\
\text { que te concentras más, como que hay mayor } \\
\text { compromiso, algo así, y en cambio cuando } \\
\text { uno no trabaja como que dices: "no tengo } \\
\text { tiempo, al ratito", o sea, siempre dices al } \\
\text { ratito, al ratito, y al fin de cuentas ni haces } \\
\text { nada (Gilberto, 2010). }\end{array}$ & $\begin{array}{l}\text { Estudiante que } \\
\text { trabaja. } \\
\text { Un estudiante que } \\
\text { trabaja muestra mayor } \\
\text { preocupación por } \\
\text { aprovechar el tiempo } \\
\text { de estudio al máximo. }\end{array}$ & $\begin{array}{l}\text { Alta valoración } \\
\text { por los estudios } \\
\text { universitarios. }\end{array}$ & $\begin{array}{l}\text { Interés por } \\
\text { tener una } \\
\text { carrera. }\end{array}$ & $\begin{array}{l}\text { Preocupación. } \\
\text { Interés académico. } \\
\text { Motivación. } \\
\text { Compromiso. } \\
\text { Responsabilidad. }\end{array}$ \\
\hline
\end{tabular}

Fuente: elaboración propia. 